\title{
Jonathan Michael Borwein 1951 - 2016: Life and Legacy
}

\author{
RICHARD P. BRENT, Mathematical Sciences Institute, Australian National University, Australia
}

\begin{abstract}
Jonathan M. Borwein (1951-2016) was a prolific mathematician whose career spanned several countries (UK, Canada, USA, Australia) and whose many interests included analysis, optimization, number theory, special functions, experimental mathematics, mathematical finance, mathematical education, and visualization. We describe his life and legacy, and give an annotated bibliography of some of his most significant books and papers.
\end{abstract}

CCS Concepts: • General and reference $\rightarrow$ Biographies.

Additional Key Words and Phrases: analysis, Borwein, experimental mathematics, mathematical education, mathematical finance, nonlinear analysis, number theory, optimization, special functions, visualization.

\section{Recommended Reference Format:}

Richard P. Brent. 2021. Jonathan Michael Borwein 1951 - 2016: Life and Legacy . Maple Trans. 1, 2, Article 14358 (November 2021), 13 pages. https://doi.org/10.5206/mt.v1i2.14358

\section{Life and Family}

Jonathan (Jon) Michael Borwein was born in St Andrews, Scotland, on 20 May 1951. He was the first of three children of David Borwein (1924-2021) and Bessie Borwein (née Flax). It was an itinerant academic family. Both Jon's father David and his younger brother Peter Borwein (1953-2020) are well-known mathematicians and occasional co-authors of Jon. His mother Bessie is a former professor of anatomy. The Borweins have an Ashkenazy Jewish background. Jon's father was born in Lithuania, moved in 1930 with his family to South Africa (where he met his future wife Bessie), and moved with Bessie to the UK in 1948. There he obtained a $\mathrm{PhD}$ (London) and then a Lectureship in St Andrews, Scotland, where Jon was born and went to school at Madras College. The family, including Jon and his two siblings, moved to Ontario, Canada, in 1963. In 1971 Jon graduated with a BA (Hons Math) from the University of Western Ontario. It was in Ontario that Jon met his future wife and lifelong partner Judith (née Roots).

When Jon won an Ontario Rhodes scholarship he returned to the UK, where he obtained an MSc (1972) and DPhil (Jesus College, Oxford, 1974). He then moved back to North America, where he held various positions at Dalhousie, CMU (Assistant/Associate Professor, 1980-82), Waterloo, and Simon Fraser. These included Professor at Dalhousie (1984-1991), Waterloo (1991-1993), and Simon Fraser (1993-2004), where he was founding Director of the Centre for Experimental and Constructive Mathematics (CECM). At the end of his time in Canada, he held a Canada Research Chair in Collaborative Technology at Dalhousie (2004-2009). At different times both Jon and his father David were presidents of the Canadian Mathematical Society.

In 2009, in the final stage of his career, Jon and Judith moved, with their daughters Naomi and Tova and grandson Jakob, to Newcastle, Australia. At the University of Newcastle he became Laureate Professor of Mathematics and founding Director of the Priority Research Centre CARMA (Computer Assisted Research in Mathematics and its Applications).

Author's address: Richard P. Brent, Mathematical Sciences Institute, Australian National University, Canberra, Australia.

Permission to make digital or hard copies of all or part of this work for any use is granted without fee, provided that copies bear this notice and the full citation on the first page. Copyrights for third-party components of this work must be honored. (C) 2021 Copyright held by the owner/author(s). Publication rights licensed to Maple Transactions, under Creative Commons CC-BY 4.0 License.

https://doi.org/10.5206/mt.v1i2.14358

Maple Trans., Vol. 1, No. 2, Article 14358. Publication date: November 2021. 


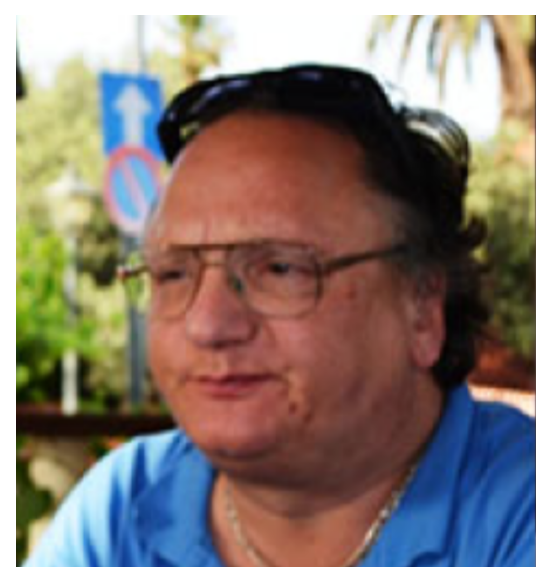

Fig. 1. Jon in Newcastle, c. 2010.

Jon had an outgoing personality, and loved to share his thoughts on mathematical (and other) topics with students and colleagues. This resulted in him having a large number of collaborators, ranging from his father and brother to his last $\mathrm{PhD}$ student. ${ }^{1}$ For more on Jon's personality, see [44] and [51, Preface].

Jon died unexpectedly on 2 August 2016, while on leave from Newcastle and on a 4-month visit to Canada as Distinguished Scholar in Residence at Western University, London, Ontario. He was survived by his wife Judith, their three daughters Naomi, Rachel and Tova, and five grandchildren, as well as by his sister Sarah, brother Peter (d. 2020), and parents David (d. 2021) and Bessie.

\section{Mathematical Legacy}

Jon was a true "polymath". While it is no longer possible for one person to be an expert in all the diverse areas of mathematics, Jon was without doubt an expert in several distinct areas, and very well-informed about many other areas of mathematics and its applications.

The areas in which Jon was an expert and made notable contributions include applied analysis, optimization, experimental mathematics, visualization, special functions, number theory, mathematical finance, and mathematical education. He was a great popularizer of mathematics, writing several books for a general mathematical audience, and giving many popular talks. The slides for some of these talks remain on his homepage [40], although, given the ephemeral nature of web sites, we do not know for how much longer they will be accessible.

Jon started his mathematical career with a 1974 DPhil in Optimization from Oxford, followed by a post-doctoral position in a strong functional analysis group in Dalhousie. His early work included papers on operations research, semi-definite programming, and integer programming. After moving to Carnegie Mellon University in 1980, he added variational analysis. In the eighties, by using his knowledge of Banach spaces, he proved some results [4] in optimization theory that were surprising as they contradicted the expert opinion of the time. Some of Jon's work in convex analysis and optimization is described in his book [27], written with his former postdoc Adrian Lewis.

Since the mid-1980s Jon was recognized as a leader in the field of nonlinear analysis. In later decades, while he added interests, he maintained activity in his earlier fields of specialization.

\footnotetext{
${ }^{1}$ An incomplete list of Jon's co-authors may be found via Google Scholar citations.
} 
Around the mid-1980s, if not earlier, Jon became "sold" on computation, aided by software such as Maple, as a tool for discovery in mathematics. In his own research he was engaged in numerically intensive optimization, primarily the use of maximum entropy methods (MEM). An early outcome was his highly cited paper [5] on the Barzilai-Borwein optimization algorithm. This motivated heuristics for minimizing functions while avoiding the heavy cost of line-search. Key to MEM is efficient numerical integration, a topic central to Jon's research in experimental mathematics, see for example [17, §4].

From 1993 to 2004, Jon collaborated with Vancouver General Hospital's Medical Imaging Group [9]. Jon's second most-cited paper [8] ${ }^{2}$ was relevant to this interaction. It provided a full treatment of the algebraic iterative methods for convex problems that are critical for turning measurements into images.

In 2004-2007, Jon, with collaborators David Bailey and others, completed his first two books $[29,30]$ on Experimental Mathematics. These books helped to popularize experimental mathematics, and were based largely on Jon's research papers. Jon continued to publish significant papers in applied experimental mathematics, often with collaborators David Bailey, David Broadhurst, and Richard Crandall. Also, when he moved to Newcastle in 2009, he continued to emphasize the importance of experimental mathematics, and founded the research centre CARMA. Up to the time of Jon's death, CARMA was successful in fostering the development of experimental mathematics within Australia. ${ }^{3}$

\subsection{Nonlinear Analysis and Optimization}

Jon's early (and some of his most significant) research was in the theory of optimization and related convex analysis. A notable paper, already mentioned above, is [5], which describes what is now known as the Barzilai-Borwein algorithm for large-scale unconstrained optimization.

Suppose that we want to approximate a stationary point of a multivariate function $F(x)$ that is differentiable in the neighbourhood of a starting point $x_{0} \in \mathbb{R}^{n}$. Several optimization methods are based on the iteration

$$
x_{k+1}=x_{k}-\gamma_{k} \nabla F\left(x_{k}\right), k \geqslant 0,
$$

but differ in their choice of "step sizes" $\gamma_{k}$. The choice used in the Barzilai-Borwein algorithm is ${ }^{4}$

$$
\gamma_{k}=\frac{\left(x_{k}-x_{k-1}\right)^{T}\left(\nabla F\left(x_{k}\right)-\nabla F\left(x_{k-1}\right)\right)}{\left\|\nabla F\left(x_{k}\right)-\nabla F\left(x_{k-1}\right)\right\|_{2}^{2}} .
$$

The motivation for this choice is that it provides a two-point approximation to the secant equation underlying quasi-Newton methods [5, §2]. This generally gives much faster convergence than the classical method of steepest descent, while having comparable cost per iteration and storage requirements.

Jon's best-known contribution to optimization theory and nonlinear analysis is perhaps his paper [4] with David Preiss, which introduces a "smooth" variational principle that has very broad applicability in problems of analysis. This extends the well-known variational principle of Ekeland [47].

\subsection{Experimental Mathematics}

Jon was a great advocate of experimental mathematics. He used advanced computer hardware and software both to discover new mathematics and to suggest, prove, or disprove, various interesting

\footnotetext{
${ }^{2}$ Citation data as at July 2021.

${ }^{3}$ As at 2021, CARMA continues without Jon, but with reduced personnel and funding.

${ }^{4}$ The paper [5] gives two different ways of choosing $\gamma_{k}$; we describe one of them here.
} 
conjectures. We give two examples here. Many other examples may be found in Jon's papers and books $[29,30,31]$.

In the first example, an experimental computation suggested a conjecture (subsequently proved) involving Euler's constant. An $n$-fold integral that arises in quantum field theory is

$$
C_{n}:=\frac{4}{n !} \int_{0}^{\infty} \cdots \int_{0}^{\infty}\left(\sum_{j=1}^{n}\left(u_{j}+u_{j}^{-1}\right)\right)^{-2} \frac{\mathrm{d} u_{1}}{u_{1}} \cdots \frac{\mathrm{d} u_{n}}{u_{n}} .
$$

In [13], Jon considered the integrals $C_{n}$, along with two similar (but slightly more complicated) integrals $D_{n}$ and $E_{n}$ that arise in mathematical physics, where the $D_{n}$ are called Ising susceptibility integrals. He obtained upper and lower bounds on the $C_{n}, D_{n}$, and $E_{n}$ integrals for large $n$, as well as closed-form expressions for $n \leqslant 4$. Some examples of the latter are $C_{4}=7 \zeta(3) / 12$ and $D_{4}=4 \pi^{2} / 9-7 \zeta(3) / 2-1 / 6$, where $\zeta$ denotes the Riemann zeta-function.

Jon showed that the $C_{n}$ integrals can be reduced to one-dimensional integrals involving the modified Bessel function $K_{0}(t)$ :

$$
C_{n}=\frac{2^{n}}{n !} \int_{0}^{\infty} t K_{0}^{n}(t) \mathrm{d} t
$$

No similar reduction is known for the $D_{n}$ or $E_{n}$ integrals.

Using the representation (1) and tanh-sinh quadrature [52] to evaluate the integrals, it was evident that the sequence $\left(C_{n}\right)$ is monotonic decreasing, with a limit, say $C_{\infty}$, given numerically by

$$
C_{\infty}=0.63047350337438679612204019271087890435458707871273 \ldots
$$

Using the Inverse Symbolic Calculator [49], Jon identified $C_{\infty}$ with a simple closed form

$$
C_{\infty}=2 e^{-2 \gamma}
$$

where $\gamma$ is Euler's constant. Finally, in [13, Theorems 1-2], Jon proved both the monotonicity of the sequence $\left(C_{n}\right)$ and convergence to the constant (2).

Some pure mathematicians might now discard all evidence of the numerical computations, and just publish the final monotonicity and convergence theorems. However, Jon recognized that such an approach would be uninformative and little short of fraudulent, since the theorems would never have been discovered without the preliminary numerical computations.

Our second example is taken from a paper [15] that Jon wrote with David Borwein (his father) and Robert Baillie. The paper gives several surprising identities (and approximate identities) involving the sinc function. We recall that $\operatorname{sinc}(x)$ is defined to be $\sin (x) / x$ if $x \neq 0$, and 1 if $x=0$.

In [15, example 1(b)], Jon asked the following question: for which positive integers $N$ does

$$
\frac{1}{2}+\sum_{n=1}^{\infty} \prod_{k=0}^{N} \operatorname{sinc}\left(\frac{n}{2 k+1}\right)=\int_{0}^{\infty} \prod_{k=0}^{N} \operatorname{sinc}\left(\frac{x}{2 k+1}\right) \mathrm{d} x ?
$$

Experimentation with Maple or Mathematica suggests that (3) is an identity for (at least) $1 \leqslant N \leqslant 6$. However, in a warning that it is not always safe to extrapolate such results, Jon showed that (3) holds for $1 \leqslant N \leqslant 40248$, but fails for all $N \geqslant 40249$, although the difference between the left-hand and right-hand sides of (3) is tiny. Several similar, though less extreme, examples may be found in Jon's papers $[10,15]$. 


\subsection{Number Theory and Special Functions}

Jon was fascinated by the constant $\pi=3.14159265358979 \cdots$, and gave many fascinating talks on this topic, some associated with an annual celebration of "pi day" on March 14th (US-style date). ${ }^{5}$ Indeed, a talk on $\pi$ is a good way to introduce some interesting mathematics to a general audience with a mathematical background, or to undergraduate students. Mathematical topics that can be motivated by $\pi$ include the concepts of irrational and transcendental numbers, rates of convergence of series and algorithms, normality of the digits of $\pi$ in decimal or binary (still an open problem), and Euler's famous relation $e^{i \pi}=-1$ that connects the constants $e, \pi, i$, and -1 .

Much of Jon's work on $\pi$ may be found in the 1987 book Pi and the AGM [25] that Jon wrote with his brother Peter. Here $A G M$ refers to the arithmetic-geometric mean of Gauss and Legendre.

In 1975-76, the present author and (independently) Salamin published the first known quadratically convergent algorithm for computing ${ }^{6} \pi$, often called the Gauss-Legendre algorithm, see for example [45]. In their book Pi and the AGM, Jon and Peter Borwein gave further quadratically (and some more rapidly) convergent algorithms. They also discussed the complexity of computing algebraic functions and elementary functions, the transcendence of $\pi$, the history of calculations of $\pi$, evaluation of various lattice sums, and many related topics. The paper [46] discusses a recentlydiscovered connection between the Gauss-Legendre algorithm and two of the Borwein algorithms for computing $\pi$.

Perhaps Jon's best-known number-theoretic result, and one in whose discovery experimental mathematics played an important role, is his paper [7] (with his brother Peter) on a cubic counterpart of Jacobi's identity, and a corresponding analogue of the arithmetic-geometric mean of Legendre and Gauss. Briefly, if $A G_{2}(a, b)$ denotes the classical arithmetic-geometric mean of $a$ and $b$, and $A G_{3}(a, b)$ denotes the Borweins' cubic analogue, then

$$
A G_{3}(1, k)={ }_{2} F_{1}\left(1 / 3,2 / 3 ; 1 ; 1-k^{2}\right)^{-1},
$$

which may be compared with Gauss's well-known identity

$$
A G_{2}(1, k)={ }_{2} F_{1}\left(1 / 2,1 / 2 ; 1 ; 1-k^{2}\right)^{-1} .
$$

Here ${ }_{2} F_{1}$ denotes a hypergeometric function. The results given in [7] have connections with the work of Ramanujan [50] on modular equations (see [6] and the review of [7] by Bruce Berndt in Math. Reviews).

\subsection{Mathematical Finance}

Although Jon was primarily a pure mathematician, his interests extended much further and included aspects of applied mathematics and statistics. This can be illustrated by his work on mathematical finance. A significant contribution is his 2014 paper [18] (with Bailey, de Prado and Zhu), provocatively titled "Pseudo-mathematics and financial charlatanism".

According to Bailey and Zhu [51, pg. 229], the paper [18] grew out of the authors' concern that, although mathematics had become a standard language to quantify financial phenomena, it was often used in a misguided fashion, lending a patina of rigour to the topic at hand, but masking some serious deficiencies. The paper demonstrated that many financial strategies and fund designs, claimed to be backed by extensive "backtests" (analyses based on historical market data), were nothing more than illusory artifacts resulting from statistical overfitting. The conclusion was that

\footnotetext{
${ }^{5}$ At the time of writing, overheads for most of these talks are available at https://www.carma.newcastle.edu.au/resources/ jon/index-talks.shtml.

${ }^{6}$ Here "computing $\pi$ " means approximating $\pi$ to any desired accuracy. For example, Archimedes found that $3 \frac{10}{71}<\pi<3 \frac{1}{7}$. It is impossible to compute the decimal or binary representation of $\pi$ exactly, since $\pi$ is transcendental [25, §11.2], so it has an infinite, nonperiodic, decimal (or binary) representation.
} 
backtest overfitting is a likely reason why so many financial strategies and fund designs fail, despite looking good on paper. The paper [18] was followed by a more detailed analysis of how to estimate the probability of backtest overfitting [21].

Jon urged his co-authors to start a blog to present the conclusions of $[18,21]$ to a broad audience. This resulted in the Mathematical Investor blog, whose mission is to identify and draw attention to abuses of mathematics and statistics in the financial field, and to call out the failure of many in the financial mathematics community for silence on these abuses [51, pg. 7].

\subsection{Mathematical Education and Visualization}

Jon had a passion for sharing his joy of mathematics with students and a more general audience. Some of his research topics were particularly well-suited for communication to high school and undergraduate students. For example, we have already mentioned his interest in experimental mathematics (\$2.2) and $\pi$ (§2.3). Jon was a keen blogger, starting this activity in 2009 when he and David Bailey founded the "Math Drudge" (now "Math Scholar") blog, ${ }^{7}$ which now has over 200 articles on a wide range of topics, covering many facets of modern mathematics, computing, and science. For a sample of the topics covered, see [51, xvii].

Jon regarded visualization as a powerful tool for both experimental mathematicians and mathematical communicators. He was fond of the maxims "it is often easier to see something than to explain it in words" and "a picture is worth a thousand words". Examples may be found in his paper [16], which contains striking images of walks in the plane associated with various mathematical constants such as $\pi, e$, and Champernowne's number $C_{4}$. In a different context, Jon used visualization to explain the dynamics of optimization algorithms such as Douglas-Rachford, for which see [22, 23].

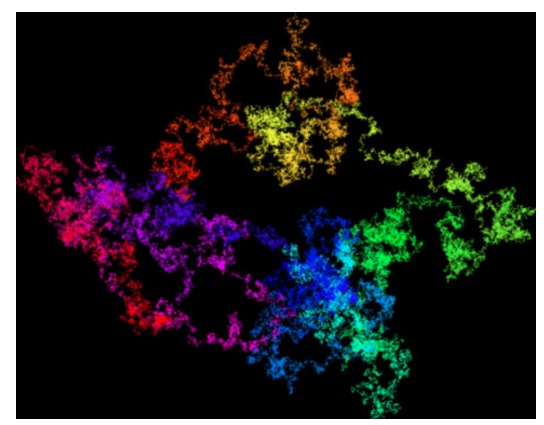

Fig. 2. A walk on the base-4 digits of $\pi$.

A high-resolution version of this image, by Francisco Aragón Artacho, is available at http: //gigapan.com/gigapans/106803.

\section{Acknowledgements}

In writing this paper I have drawn heavily on information provided in the JBCC Conference Proceedings by Brailey Sims et al. [51], and the obituaries by David Bailey [37] and by Judith Borwein, Naomi Borwein, and Brailey Sims [44]. I have also made use of the extensive list of Jon's publications maintained by David Bailey and Nelson Beebe [39], and information provided on the websites [40, 41, 42, 53]. Thanks go to Heinz Bauschke, Tony Guttmann, Judy-anne Osborn, and Brailey Sims, for helpful comments on previous drafts.

${ }_{7 \text { https://www.mathscholar.org. }}$ 


\section{Annotated bibliography}

An online list of Jon's publications is available at [39]. It contains over 2, 000 items (although some are essentially duplicates), including (at least) 387 papers in refereed journals, 103 refereed or invited conference proceedings and book chapters, and 26 books. We list only a small sample, which includes Jon's better-known books and most influential papers.

In the following, papers [1]-[23] include some of Jon's most influential or highly cited papers. The list includes papers that were given by Jon himself as "ten career-best publications" (up to mid-2013) in an ARC grant application, and the comments on these papers are based on Jon's own. Other papers have been selected by the author.

Jon wrote at least 26 books. A few, like [25], are major research monographs. Some, like [27], are advanced textbooks. Others are "dictionaries" (e.g. [24, 26]) or edited collections of papers by various authors on a particular topic (e.g. [36]). Last but not least, there are some significant "popular" books written for a general mathematical audience, e.g. those such as [31] designed to popularize experimental mathematics. We have included at least one from each category in the list [24]-[36] below.

Finally, at the end, we give other relevant sources [37]-[53].

The papers and books by Jon are ordered chronologically by date of publication; the final section is ordered alphabetically by author.

\section{Selected papers by Jonathan Borwein}

[1] J. M. Borwein, Proper efficient points for maximization with respect to cones, SIAM f. Control and Optimization 15, 1 (1977), 57-63.

In this paper Jon gave a subtle geometric accounting of Pareto efficiency that allowed for application in infinite-dimensional and conic settings.

[2] J. M. Borwein and P. B. Borwein, The arithmetic-geometric mean and fast computation of elementary functions, SIAM Review 26, 3 (1984), 351-366.

This review summarized and helped to popularize the work of Brent and Salamin on fast AGMbased algorithms for the computation of elementary functions, and outlined the historical foundations of these algorithms in the much earlier work of Gauss, Legendre, and King. It also presented a new, quadratically convergent, algorithm for computing $\pi$. The paper [2] laid the foundations for the 1987 book Pi and the AGM [25], see §2.3.

[3] J. M. Borwein, Stability and regular points of inequality systems, f. Optimization Theory and Applications 48, 1 (1986), 9-52.

This paper exemplifies Jon's skill with delicate technical arguments. It introduced a notation of metric regularity that has been exploited by many researchers.

[4] J. M. Borwein and D. Preiss, A smooth variational principle with application to subdifferentiability and to differentiability of convex functions, Trans. Amer. Math. Soc. 303, 2 (1987), 517-527.

This is a well-known paper in optimization theory. It provided a result that was previously thought to be impossible, and that has since become a work-horse in non-smooth analysis.

[5] J. Barzilai and J. M. Borwein, Two-point step size gradient methods, IMA f. of Numerical Analysis 8, 1 (1988), 141-148.

Although ignored for ten years, this paper is now Jon's most highly cited (as at July 2021), and is the basis for much current work, partly because it is suited to large-scale, parallel optimization. It describes what is now known as the Barzilai-Borwein algorithm for large-scale unconstrained optimization. See $§ 2.1$ above for further details. 
[6] J. M. Borwein, P. B. Borwein, and D. H. Bailey, Ramanujan, modular equations, and approximations to pi or how to compute one billion digits of pi, Amer. Math. Monthly 96, 3 (March 1989), 201-219.

This paper won the 1993 Chauvenet $^{8}$ and Hasse ${ }^{9}$ prizes for expository writing. It rapidly covers various topics related to $\pi$. In particular, it describes some of Ramanujan's rapidly converging series for $1 / \pi$, and explains their derivation using modular equations. It also describes the Borwein-Borwein quartically convergent algorithm for $\pi$, and some historical computations of $\pi$. The title is slightly misleading, as the authors did not compute $10^{9}$ digits of $\pi$, they merely showed that such a computation was feasible. In fact, Bailey computed 29 million digits of $\pi$ in 1986 . The first computation of $10^{9}$ digits of $\pi$ was performed by Kanada and Tamura in November 1989.

[7] J. M. Borwein and P. B. Borwein, A cubic counterpart of Jacobi's identity and the AGM, Trans. Amer. Math. Soc. 323, 2 (1991), 691-701. MR1010408 (91e:33012).

This is one of Jon's first papers in which experimental mathematics played a critical role. The cubic analogue of Jacobi's identity, obtained with Peter Borwein, is perhaps Jon's best-known number-theoretic result. ${ }^{10}$

[8] H. H. Bauschke and J. M. Borwein, On projection algorithms for solving convex feasibility problems, SIAM Review 38, 3 (1996), 367-426.

This paper, one of Jon's most highly cited, grew out of a prize-winning $\mathrm{PhD}$ thesis supervised by Jon, and has become the basic resource for a large subject.

[9] H. H. Bauschke, D. Noll, A. Celler, and J. M. Borwein, An EM algorithm for dynamic SPECT, IEEE Trans. Med. Imaging 18, 3 (1999), 252-261.

This paper describes some of Jon's work related to medical imaging.

[10] D. Borwein and J. M. Borwein, Some remarkable properties of sinc and related integrals, Ramanujan 7. 5, 1 (2001), 73-89.

One of several papers papers written by Jon and his father David on surprising properties of the "sinc" function $\operatorname{sinc}(x):=\sin (x) / x$. See also $\$ 2.2$ above. For another example, see [15].

[11] J. M. Borwein, D. M. Bradley, D. J. Broadhurst, and P. Lisonĕk, Special values of multiple polylogarithms, Trans. Amer. Math. Soc. 353, 3 (2001), 907-941. MR1709772 (2003i:33003).

Special values of the Riemann zeta-function and Euler sums have been the subject of much experimentation and several conjectures. This paper succeeds in proving some of these comjectures, including one of Don Zagier. This is also a paper where Jon uses one of his favourite tools - the PSLQ algorithm of Ferguson et al. [48]. As Patrick D. F. Ion wrote at the end of a long review in Math. Reviews, "this paper is an invitation to a very rich field of mathematics and a review can only touch on some of its content".

[12] J. M. Borwein, Maximal monotonicity via convex analysis, f. of Convex Analysis 13, 3 (2006), 561-586.

This was the basis for a comprehensive chapter on monotone operators in Jon's 2010 book Convex Functions [32], in which he derived most of the (originally very hard) theory entirely within convex analysis.

[13] D. H. Bailey, J. M. Borwein, and R. E. Crandall, Integrals of the Ising class, f. Phys. A: Math. Gen. 39 (2006), 12271-12302.

An analysis of three classes of integrals that arise in mathematical physics. See $\S 2.2$ for further details.

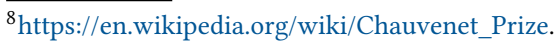

${ }^{9}$ https://en.wikipedia.org/wiki/Merten_M._Hasse_Prize.

${ }^{10}$ There is a typographical error in $[7$, Theorem $2.1(\mathrm{~b})]$, where " $A G_{2}$ " should be " $A G_{3}$ ".
} 
[14] D. H. Bailey, J. M. Borwein, D. J. Broadhurst, and M. L. Glasser, Elliptic integral representation of Bessel moments and applications, f. Phys. A: Math. Theory 41, 20 (2008), 205203. https: //doi.org/10.1088/1751-8113/41/20/205203.

A referee wrote: "a major breakthrough in our understanding of ... massive amplitudes in perturbative quantum field theory and condensed matter physics. It is an astonishing exemplar of interdisciplinary work."

[15] R. Baillie, D. Borwein, and J. M. Borwein, Surprising sinc sums and integrals, Amer. Math. Monthly 115, 10 (2008), 888-901.

One of several papers papers by Jon on properties of the "sinc" function $\operatorname{sinc}(x):=\sin (x) / x$. See also [10].

[16] F. J. Aragón Artacho, D. H. Bailey, J. M. Borwein, and P. B. Borwein, Walking on real numbers, The Mathematical Intelligencer 35, 1 (2013), 42-60.

This visualization and analytic study was a popular success, picked up by Wired, Gizmodo and other technology magazines. It was a finalist in the 2013 NSF International Scientific Visualization Contest.

[17] J. M. Borwein and R. E. Crandall, Closed forms: what they are and why we care, Notices Amer. Math. Soc. 60, 1 (2013), 50-65.

A discussion of the mathematical concept of "closed form", including recent examples where the question of closure is both important and elusive.

[18] D. H. Bailey, J. M. Borwein, M. L. de Prado, and Q. J. Zhu, Pseudo-mathematics and financial charlatanism: the effects of backtest overfitting on out-of-sample performance, Notices Amer. Math. Soc. 61, 5 (2014), 458-471.

This paper demonstrated that many financial strategies and fund designs are based on nothing more than illusory artifacts resulting from statistical overfitting. See also §2.4.

[19] J. M. Borwein, I prefer pi: a brief history and anthology of articles in the American Mathematical Monthly, Amer. Math. Monthly 122, 3 (2015), 195-216.

Presents a historical overview of algorithms for computing $\pi$, from Archimedes to BrentSalamin, the Borwein quartic algorithm, spigot algorithms, infinite product formulas, and the curious behaviour of erroneous digits in the Gregory series for $\pi$.

[20] D. H. Bailey, J. M. Borwein, R. P. Brent, and M. Reisi, Reproducibility in computational science: a case study: randomness of the digits of pi, Experimental Mathematics 22, 3, (2017), 298-305. This paper, whose final version was sent for publication just a few days before Jon's death, covers two of Jon's major interests: the importance of reproducibility of published computational results, and the question of normality (roughly speaking, randomness) of the decimal digits of mathematical constants such as $\pi$. A paper by Ganz in the same journal had claimed that a certain statistical test showed that the digits of $\pi$ were significantly non-random. Jon's response [20] pointed out the difficulties in reproducing Ganz's results, and reached the opposite conclusion, i.e. that there was no significant statistical evidence for nonrandomness of the digits of $\pi$.

[21] D. H. Bailey, J. M. Borwein, M. L. de Prado, and Q. J. Zhu, The probability of backtest overfitting, f. Computational Finance 20, 4 (2017), 39-69.

A sequel to [18], giving a detailed analysis of how to estimate the probability of backtest overfitting.

[22] J. M. Borwein and M. K. Tam, Reflection methods for inverse problems with applications to protein conformation determination, in Generalized Nash Equilibrium Problems, Bilevel Programming and MPEC, D. Aussel and C. Lalitha (eds.), Forum for Interdisciplinary Mathematics, Springer, 2017, 83-100. https://doi.org/10.1007/978-981-10-4774-9_5. Also arXiv:1408.4213. 
An interdisciplinary paper on the application of the Douglas-Rachford method to protein conformation determination, written by Jon and one of his last $\mathrm{PhD}$ students, Matt Tam.

[23] J. M. Borwein, S. B. Lindstrom, B. Sims, A. Schneider, and M. P. Skerritt, Dynamics of the Douglas-Rachford method for ellipses and p-spheres, Set-Valued Var. Anal. 26, 2 (2018), 385-403. https://doi.org/10.1007/s11228-017-0457-0.

A good illustration of how the maxim "A picture is worth 1000 words" can be applied to a serious topic - the properties of an optimization algorithm. Jon's co-authors include his last PhD student, Matt Skerritt.

\section{Selected books by Jonathan Borwein}

[24] E. J. Borowski and J. M. Borwein, Dictionary of Mathematics, Collins, Glasgow, 1989, 675+xiv pp. Reprinted 1989, 1991. Also Arabic (1991), Chinese (1995), Indonesian (1995), and Italian (1995) editions. Second (revised) edition 2002, 631+ix pp.

A useful dictionary of mathematical terms and concepts.

[25] J. M. Borwein and P. B. Borwein, Pi and the AGM: A Study in Analytic Number Theory and Computational Complexity, John Wiley, New York, 1987, 414+xv pp. Reprinted 1988, 1996. Chinese edition 1995, paperback 1998.

This book, by Jon and his brother Peter Borwein, is a classic work on asymptotically fast algorithms for computing $\pi$ and various elementary and special functions by methods related to the arithmetic-geometric mean of Gauss and Legendre (and much more). See also §2.3 and [28].

[26] J. M. Borwein and P. B. Borwein, A Dictionary of Real Numbers, Wadsworth and Brooks/Cole Advanced Books and Software, Pacific Grove, California, 1990, 424+viii pp.

An early attempt to list "interesting" real numbers in a searchable format, later made obsolete by software such as the Inverse Symbolic Calculator [49] developed by Simon Plouffe, Jon, and others.

[27] J. M. Borwein and A. S. Lewis, Convex Analysis and Nonlinear Optimization: Theory and Examples, Springer-Verlag, New York, 2000, $\mathrm{x}+273 \mathrm{pp}$.

A highly-cited text on convex optimization.

[28] L. Berggren, J. M. Borwein, and P. B. Borwein (editors), Pi: A Source Book, third ed., Springer, 2004, $\mathrm{xx}+797 \mathrm{pp}$.

Jon was fascinated by the constant $\pi$ and gave many popular talks on it, usually on "Pi Day" (March 14th, or 3.14 if written in the north American manner).

This book documents the history of $\pi$ from the dawn of mathematical time to the present. One of the beauties of the literature on $\pi$ is that it allows for the inclusion of very modern, yet accessible, mathematics. The articles on $\pi$ in this collection fall into three classes. First there is a selection from the mathematical and computational literature of four millennia. Second is a variety of historical studies on the cultural significance of the number. Finally, there is a selection of pieces that are anecdotal, fanciful, or simply amusing. There is a substantial exposition of the recent history of the computation of digits of $\pi$, the normality of the distribution of the digits (still an open problem), and new translations of works by Viète and Huygens.

Jon once remarked that the difficulty in producing such a collection lay mainly in tracking down and obtaining permissions from all the copyright holders.

[29] J. M. Borwein and D. Bailey, Mathematics by Experiment: Plausible Reasoning in the 21st Century, 2nd edition, A K Peters, Wellesley, Mass., 2008, xii+377 pp. (First edition, 2004, x+288 pp.)

[30] J. M. Borwein, D. Bailey, N. Calkin, R. Girgensohn, R. Luke, and V. Moll, Experimental Mathematics in Action, A K Peters, Wellesley, Mass., 2007, xii+322 pp. 
In the two books [29, 30], Jon and his co-authors explain experimental mathematics in a lively, accessible fashion, and give many engaging examples of this "new paradigm" in action.

[31] J. M. Borwein and K. Devlin, The Computer as Crucible: an Introduction to Experimental Mathematics, CRC Press, Boca Raton, Florida, 2008, xi+150 pp.

Another book along the lines of $[29,30]$, covering a variety of topics and examples in the field of experimental mathematics, but at a more introductory level. Jon was a leader in promoting experimental mathematics via his talks, papers and books.

[32] J. M. Borwein and J. D. Vanderwerff, Convex Functions: Constructions, Characterizations and Counterexamples, Cambridge University Press, Cambridge, UK, 2010, x+521 pp.

A research monograph on convex functions, including much previously unpublished research by Jon. See [12] above.

[33] J. M. Borwein and M. Skerritt, An Introduction to Modern Mathematical Computing with Maple, Springer Undergraduate Series in Mathematics and Technology (SUMAT), 2011, xvi+216 pp.

An undergraduate-level text on techniques for mathematical computation, illustrated using Maple, by Jon and his student Matthew Skerritt. Covers elementary number theory, calculus, introductory linear algebra, visualization, and interactive geometric computation.

[34] J. M. Borwein, M. L. Glasser, R. C. McPhedran, J. G. Wan, and I. J. Zucker, Lattice Sums Then and Now, Encyclopedia of Mathematics and its Applications, Vol. 150, Cambridge University Press, 2013, xx+368 pp.

This is a comprehensive overview of lattice sums and their applications. Jon and his co-authors also provide commentaries on open questions, and explain modern techniques that simplify the task of finding new results in this fascinating and ongoing field. Although primarily a survey, much of the authors' research is included.

[35] J. M. Borwein, A. van der Poorten, J. Shallit, and W. Zudilin, Neverending Fractions, Australian Mathematical Society Lecture Series, Vol. 23, Cambridge University Press, 2014, ix+212 pp. A book on various aspects of continued fractions. Despite their classical nature, continued fractions are a neverending research area, with a body of results accessible enough to suit a wide audience. This book brings these results together and offers some fresh perspectives. Topics include elementary and metric properties, quadratic irrationals, and more exotic topics such as folded continued fractions and Somos sequences. There are also some surprising applications of the theory to seemingly unrelated problems in number theory.

[36] D. H. Bailey and J. M. Borwein (editors), Pi the Next Generation. A Sourcebook on the Recent History of Pi and its Computation, Springer-Verlag, Berlin, 2016, xiv+507 pp.

A continuation and update of [28], including 25 papers dated 1976-2015.

\section{Other references}

[37] D. H. Bailey, Jonathan Borwein dies at 65, http://experimentalmath.info/blog/2016/08/jonathanborwein-dies-at-65/, 2 August 2016, accessed 12 July 2021.

A brief obituary by Jon's collaborator of 31 years, published soon after Jon's death, on a blog to which Jon had been a regular contributor.

[38] D. H. Bailey et al. (editors), Proceedings of a Workshop on Computational and Analytical Mathematics in honour of Jonathan Borwein's 60th birthday, Springer Proceedings in Mathematics and Statistics, Vol. 50, 2013.

The Proceedings of a Workshop held at Simon Fraser University, in May 2011, to celebrate Jon's 60th birthday. Contains 31 contributed papers on topics of interest to Jon.

[39] D. H. Bailey and N. Beebe, Publications and talks by (and about) Jonathan M. Borwein, https: //www.jonborwein.org/jmbpapers/, accessed 12 July 2021. 
This gives the most complete and up-to-date list of Jon's talks and publications, containing over 2,000 items.

[40] Jonathan Borwein homepage, https://www.carma.newcastle.edu.au/jon/, accessed 12 July 2021.

Jon's CARMA homepage, archived as it was at the time of his death.

[41] Jonathan Borwein Memorial website, https://jonborwein.org, accessed 12 July 2021.

Contains information about Jon and events that were held in his memory in Canberra, Jerusalem, Newcastle, Niagara Falls, and Paris, in the period 2016-2017 after his death.

[42] Jonathan M. Borwein Commemorative Conference website, https://carma.newcastle.edu.au/ meetings/jbcc/, accessed 12 July 2021.

The website for the commemorative conference whose Proceedings were later published by Springer [51]. Includes abstracts and (in some cases) slides from the talks.

[43] Jonathan Michael Borwein, Mathematics Genealogy, https://www.genealogy.math.ndsu.nodak. edu/id.php?id=23187.

Lists Jon's $16 \mathrm{PhD}$ students from 1984 to 2020, as well as his academic "ancestors".

[44] Judith Borwein, Naomi Borwein, and Brailey Sims, fonathan M. Borwein FAAAS, FBAS, FAustMS, FAA, FAMS, FRSNSW 20 May 1951 to 2 August 2016, Gazette AustMS, November 2017, 289-293, https://www.austms.org.au/Publ/Gazette/2017/Nov17/ObitBorwein.pdf, accessed 12 July 2021.

A brief obituary of Jon in the Gazette of the Australian Mathematical Society.

[45] Richard Brent, Old and new algorithms for $\pi$, Notices of the AMS 60, 1 (2013), 7. http://www. ams.org/notices/201301/rnoti-p7.pdf.

Gives some historical comments on algorithms for rapidly computing $\pi$ and, more generally, elementary functions, via the AGM and fast multiplication.

[46] Richard Brent, The Borwein brothers, $\pi$ and the AGM, in [51], 323-348.

Discusses in detail some aspects of the book [25] and proves that the Gauss-Legendre algorithm for computing $\pi$ is equivalent to the Borwein-Borwein quartic algorithm.

[47] Ivar Ekeland, On the variational principle, J. Math. Anal. Appl. 47 (1974), 324-353.

Gives a useful variational principle that was a starting point for Jon's paper [4].

[48] H. R. P. Ferguson, D. H. Bailey, and S. Arno, Analysis of PSLQ, an integer relation finding algorithm, Math. Comp. 68 (1999), 351-369.

One of Jon's favorite algorithms, which he often used to discover surprising identities, many of which have subsequently been proved by Jon and others. Jon's last PhD student, Matt Skerritt, studied the problem of generalising PSLQ to algebraic integer relations.

[49] Simon Plouffe et al., Inverse Symbolic Calculator, http://wayback.cecm.sfu.ca/projects/ISC/ ISCmain.html, accessed 12 July 2021.

Useful software for "reverse engineering" mathematical constants that may be expressed in terms of well-known constants such as $\pi, e, \gamma$, etc. See the comments on [26].

[50] Srinivasa Ramanujan, Modular equations and approximations to $\pi$, Quart. f. Math. 45 (1914), 350-372.

Gives some of Ramanujan's results on modular equations and the first examples of RamanujanSato formulæ for $1 / \pi$. See also [6].

[51] Brailey Sims et al. (editors), From Analysis to Visualisation: A Celebration of the Life and Legacy of Jonathan M. Borwein, Callaghan, Australia, September 2017. Springer Proceedings in Mathematics and Statistics, Vol. 313, 2020, xxi + 439 pp. https://doi.org/10.1007/978-3-03036568-4.

This volume includes a Preface and 23 papers, on topics of interest to Jon, by his former students and colleagues. See also [42]. A similar but earlier volume is [38]. 
[52] H. Takahasi and M. Mori, Double exponential formulas for numerical integration, Publ. RIMS, Kyoto Univ. 9 (1974), 721-741.

The first paper on tanh-sinh quadrature, often used by Jon and his collaborators to accurately evaluate integrals which could then be investigated using the PSLQ algorithm [48] or the Inverse Symbolic Calculator ([49], §2.2).

[53] Wikipedia, Jonathan Borwein, https://en.wikipedia.org/wiki/Jonathan_Borwein, accessed 12 July 2021.

Jon's Wikipedia entry. 